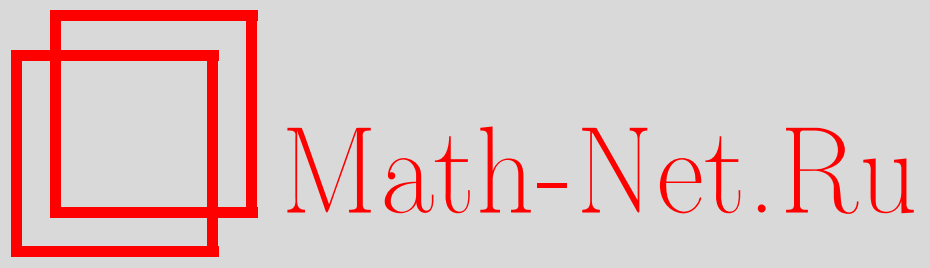

Е. В. Бурнаев, Е. А. Файнберг, А. Н. Ширяев, Об асимптотической оптимальности второго порядка в минимаксной задаче скорейшего обнаружения момента изменения сноса у броуновского движения, Теория вероятн. и ее примен., 2008, том 53, выпуск $3,557-575$

DOI: https://doi.org/10.4213/tvp2449

Использование Общероссийского математического портала Math-Net.Ru подразумевает, что вы прочитали и согласны с пользовательским соглашением

http://www . mathnet.ru/rus/agreement

Параметры загрузки:

IP : 54.162 .127 .20

26 апреля 2023 г., 14:07:30

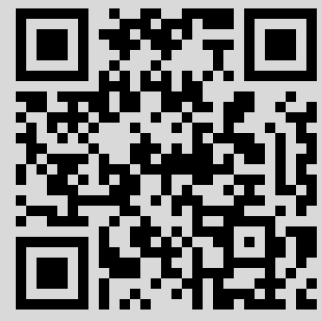


(C) 2008 г. БУРНАЕВ Е. В.*, ФАЙНБЕРГ Е. А.

\section{ОБ АСИМПТОТИЧЕСКОЙ ОПТИМАЛЬНОСТИ ВТОРОГО ПОРЯДКА В МИНИМАКСНОЙ ЗАДАЧЕ СКОРЕЙШЕГО ОБНАРУЖЕНИЯ МОМЕНТА ИЗМЕНЕНИЯ СНОСА У БРОУНОВСКОГО ДВИЖЕНИЯ ${ }^{1)}$}

В задаче о «разладке», состоящей в появлении сноса у броуновского движения в момент $\theta \geqslant 0$, исследуются минимаксные риски $C(T)=\inf _{\tau \in \mathfrak{M}_{T}} \sup _{\theta} \mathbf{E}_{\theta}(\tau-\theta \mid \tau \geqslant \theta)$ и $\bar{C}(T)=\inf _{\bar{\tau} \in \overline{\mathfrak{M}}_{T}} \sup _{\theta} \mathbf{E}_{\theta}(\bar{\tau}-$ $\theta \mid \bar{\tau} \geqslant \theta)$, где $\mathfrak{M}_{T}$ - класс моментов остановки $\tau$ таких, что $\mathbf{E}_{\infty} \tau=T$, и $\overline{\mathfrak{M}}_{T}$ - класс рандомизированных моментов остановки $\bar{\tau}$ таких, что $\mathbf{E}_{\infty} \bar{\tau}=T$. Основные результаты работы относятся к получению для этих рисков оценок сверху и снизу, из которых, в частности, вытекает существование моментов, обладающих при $T \rightarrow \infty$ свойством асимптотической оптимальности первого и второго порядка (соответственно для $C(T)$ и $\bar{C}(T))$.

Ключевые слова и фразы: задача о «разладке», броуновское движение, минимаксный риск, асимптотическая оптимальность первого и второго порядка.

\section{1. Введение. Постановка задачи и обсуждение результатов}

1.1. Байесовская формулировка задачи скорейшего обнаружения момента изменения сноса у броуновского движения («задача о разладке») была дана в [6], [7]. Там же было найдено решение этой задачи для экспоненциального априорного распределения момента появления разладки и специальным предельным переходом было получено решение

* Институт проблем передачи информации им. А. А. Харкевича РАН, Б. Каретный пер., 19, 127994 Москва, Россия; e-mail: burnaev@iitp.ru

** State University of New York at Stony Brook, Stony Brook, NY, 11794-3600, USA; e-mail: Eugene.Feinberg@sunysb.edu

*** Математический институт им. В. А. Стеклова РАН, ул. Губкина, 8, 119991 Москва, ГСП-1, Россия; e-mail: albertsh@mi.ras.ru

1) Первый автор частично поддержан аналитической ведомственной целевой программой РНП.2.2.1.1.2467, грантами НШ-5379.2006.1, РГНФ 06-02-91821 а/G, программой фундаментальных исследований Президиума РАН 15. Второй и третий авторы поддержаны грантами NSF (DMI-0300121 и DMI-0600538) и NYSTAR, а также грантами РФФИ 08-01-00740 и 08-01-91205-ЯФ. 
задачи о разладке в обобщенной байесовской постановке (с «равномерным» распределением на $\left.\mathbf{R}^{+}=[0, \infty)\right)$, к которой сводится задача о скорейшем обнаружении нарушения стационарного режима (см. [7], [21, п. 4.2], [22, § 3.9]).

Прямое решение обобщенной байесовской задачи - без предельного перехода - было приведено в работе [11] (вариант (В)), где были также получены (см. [11, теорема 3.1]) оценки сверху и снизу для минимаксного риска, характеризуюшего степень запаздывания в обнаружении момента появления разладки (вариант $(\mathrm{C})$ ).

$\mathrm{C}$ целью уменьшения «зазора» между верхней и нижней границами для минимаксного риска (см. неравенства (13) ниже и неравенства (3.1) в [11]) в настоящей работе рассматривается возможность использования (для подачи сигнала о появлении разладки) не только марковских моментов (моментов остановки), определяемых ходом наблюдаемого процесса, но и рандомизированных моментов (вариант $(\overline{\mathrm{C}})$ ).

Основной результат данной работы состоит в доказательстве того, что в классе рандомизированных моментов существует момент, являющийся асимптотически оптимальным второго порядка при $T \rightarrow \infty$; см. неравенства (14) в п. 1.6. (Для этого рандомизированного момента оценки сверху и снизу соответствующего минимаксного риска дают меньший «зазор», нежели при использовании лишь нерандомизированных моментов. При этом первые два члена в этих оценках совпадают, а остальные стремятся к нулю при $T \rightarrow \infty$.)

1.2. Постановка задач о разладке в вариантах $(\mathrm{B}),(\overline{\mathrm{B}}),(\mathrm{C})$ и $(\overline{\mathrm{C}})$ состоит в следующем (ср. с [11]).

Пусть $\left(\Omega, \mathscr{F},\left(\mathscr{F}_{t}\right)_{t \geqslant 0}, \mathbf{P}\right)$ - фильтрованное вероятностное пространство и $B=\left(B_{t}\right)_{t \geqslant 0}$ - стандартное броуновское движение $\left(\mathbf{E} B_{t}=0\right.$, $\left.\mathbf{E} B_{t}^{2}=t\right)$.

Предполагается, что наблюдаемый процесс $X=\left(X_{t}\right)_{t \geqslant 0}$ имеет следующую структуру:

$$
X_{t}=\mu(t-\theta)^{+}+\sigma B_{t},
$$

где $x^{+}=\max (x, 0), \mu \neq 0, \sigma>0$ и $\theta$ - параметр, принимающий значения в $[0, \infty]$ и интерпретируемый как момент возникновения разладки. Поскольку, согласно (1),

$$
d X_{t}= \begin{cases}\sigma d B_{t}, & t<\theta, \\ \mu d t+\sigma d B_{t}, & t \geqslant \theta\end{cases}
$$

то в момент $\theta$ меняется снос у броуновского движения (происходит «разладка»).

Обозначим $\mathbf{P}_{\theta}=\operatorname{Law}(X \mid \mathbf{P}, \theta)$ - распределение вероятностей (в пространстве непрерывных функций $\mathrm{C}=\mathrm{C}[0, \infty)$ с борелевской $\sigma$-алгеброй $\mathscr{C}$ ) процесса $X$ в предположении, что разладка произошла в мо- 
мент $\theta$. В таком случае распределение $\mathbf{P}_{\infty}$ - это распределение вероятностей процесса $X$, когда $\theta=\infty$ (и, значит, $\left.\mathbf{P}_{\infty}=\operatorname{Law}\left(\sigma B_{t}, t \geqslant 0\right)\right)$; распределение $\mathbf{P}_{0}$ - это распределение вероятностей процесса $X$ в предположении, что разладка имеет место в момент $\theta=0$ (и, значит, $\left.\mathbf{P}_{0}=\operatorname{Law}\left(\mu t+\sigma B_{t}, t \geqslant 0\right)\right)$.

$\mathrm{C}$ целью упрощения обозначений будем предполагать (и это не ограничивает общности), что в исходном фильтрованном вероятностном пространстве поток $\left(\mathscr{F}_{t}\right)_{t \geqslant 0}$ совпадает с $\left(\mathscr{F}_{t}^{B}\right)_{t \geqslant 0}$, где $\mathscr{F}_{t}^{B}=\sigma\left(B_{s}, s \leqslant t\right)$ суть $\sigma$-алгебры, порожденные броуновским движением. Отметим, что $\left(\mathscr{F}_{t}^{B}\right)_{t \geqslant 0}=\left(\mathscr{F}_{t}^{X}\right)_{t \geqslant 0}$ при любом $\theta \in[0, \infty]$.

Пусть $\mathfrak{M}=\{\tau: \tau=\tau(\omega)<\infty, \omega \in \Omega\}-$ класс конечных марковских моментов (моментов остановки) относительно потока $\left(\mathscr{F}_{t}\right)_{t \geqslant 0}$ $\left(=\left(\mathscr{F}_{t}^{B}\right)_{t \geqslant 0}\right)$.

Помимо класса $\mathfrak{M}$ (нерандомизированных моментов остановки) введем более широкий класс рандомизированных моментов остановки $\bar{\tau}=\bar{\tau}(\bar{\omega})$, определяемых следующим образом (ср. с $[8$, гл. II, $\S 7])$.

Пусть $\widetilde{\Omega}=[0,1], \widetilde{\mathscr{F}}=\mathscr{B}([0,1])-$ борелевская система множеств на $\widetilde{\Omega}$ и $\widetilde{\mathbf{P}}=\lambda$ - лебеговская мера на $(\widetilde{\Omega}, \widetilde{F})$. Введем новое фильтрованное вероятностное пространство $\left(\bar{\Omega}, \overline{\mathscr{F}},\left(\overline{\mathscr{F}}_{t}\right)_{t \geqslant 0}, \overline{\mathbf{P}}\right)$ с $\bar{\Omega}=\Omega \times \widetilde{\Omega}$, $\overline{\mathscr{F}}=\mathscr{F} \otimes \widetilde{\mathscr{F}}, \overline{\mathscr{F}}_{t}=\mathscr{F}_{t} \otimes \widetilde{\mathscr{F}}, \overline{\mathbf{P}}=\mathbf{P} \otimes \widetilde{\mathbf{P}}$.

Полагая $\bar{\omega}=(\omega, \widetilde{\omega})$, будем говорить, что моменты $\bar{\tau}=\bar{\tau}(\bar{\omega})$ являются рандомизированными марковскими моментами (моментами остановки), если $\{\bar{\omega}: \bar{\tau}(\bar{\omega}) \leqslant t\} \in \overline{\mathscr{F}}_{t}$ для каждого $t \geqslant 0$.

Класс таких конечных рандомизированных моментов будем обозначать $\overline{\mathfrak{M}}=\{\bar{\tau}: \bar{\tau}=\bar{\tau}(\bar{\omega})<\infty, \bar{\omega} \in \bar{\Omega}\}$.

Условимся также о следующих обозначениях. Если $Y=Y(\omega)$ и $\widetilde{Y}=\tilde{Y}(\widetilde{\omega})$ - случайные элементы, заданные на $(\Omega, \mathscr{F}, \mathbf{P})$ и $(\widetilde{\Omega}, \widetilde{\mathscr{F}}, \widetilde{\mathbf{P}})$ соответственно, то они естественным образом могут рассматриваться и как элементы на $(\bar{\Omega}, \overline{\mathscr{F}}, \overline{\mathbf{P}})$, если полагать $\bar{Y}(\bar{\omega})=Y(\omega)$ и $\overline{\tilde{Y}}(\bar{\omega})=\tilde{Y}(\widetilde{\omega})$ для $\bar{\omega}=(\omega, \widetilde{\omega})$.

По смыслу рассматриваемых далее задач скорейшего обнаружения моменты $\tau$ и $\bar{\tau}$ будут интерпретироваться как (нерандомизированные и рандомизированные) моменты подачи «сигнала» о появлении разладки.

1.3. Пусть для всякого $T>0$

$$
\mathfrak{M}_{T}=\left\{\tau \in \mathfrak{M}: \mathbf{E}_{\infty} \tau=T\right\}, \quad \overline{\mathfrak{M}}_{T}=\left\{\bar{\tau} \in \overline{\mathfrak{M}}: \overline{\mathbf{E}}_{\infty} \bar{\tau}=T\right\}
$$

где $\mathbf{E}_{\infty}$ и $\overline{\mathbf{E}}_{\infty}$ - усреднение по мерам $\mathbf{P}_{\infty}$ и $\overline{\mathbf{P}}_{\infty}=\mathbf{P}_{\infty} \otimes \widetilde{\mathbf{P}}$ соответственно. По своему смыслу класс $\mathfrak{M}_{T}$ - это класс тех моментов $\tau \in \mathfrak{M}$, для которых среднее время $\mathbf{E}_{\infty} \tau$, т.е. среднее время до подачи сигнала о наличии разладки, если ее на самом деле нет, равно $T$. Попросту говоря, $\mathbf{E}_{\infty} \tau$ - это среднее время до объявления «ложной тревоги» о наличии разладки в ходе наблюдаемого процесса. 
Введем для $T>0$ следующие величины, характеризующие качество моментов остановки из классов $\mathfrak{M}_{T}$ и $\overline{\mathfrak{M}}_{T}$ :

$$
B(T)=\inf _{\tau \in \mathfrak{M}_{T}} \frac{1}{T} \int_{0}^{\infty} \mathbf{E}_{\theta}(\tau-\theta)^{+} d \theta, \quad \bar{B}(T)=\inf _{\bar{\tau} \in \overline{\mathfrak{M}}_{T}} \frac{1}{T} \int_{0}^{\infty} \overline{\mathbf{E}}_{\theta}(\bar{\tau}-\theta)^{+} d \theta,
$$

где, напомним, $x^{+}=\max (x, 0)$, и

$$
C(T)=\inf _{\tau \in \mathfrak{M}_{T}} \sup _{\theta \geqslant 0} \mathbf{E}_{\theta}(\tau-\theta \mid \tau \geqslant \theta), \quad \bar{C}(T)=\inf _{\bar{\tau} \in \overline{\mathfrak{M}}_{T}} \sup _{\theta \geqslant 0} \overline{\mathbf{E}}_{\theta}(\bar{\tau}-\theta \mid \bar{\tau} \geqslant \theta) .
$$

Величины $\int_{0}^{\infty} \mathbf{E}_{\theta}(\tau-\theta)^{+} d \theta$ и $\int_{0}^{\infty} \overline{\mathbf{E}}_{\theta}(\bar{\tau}-\theta)^{+} d \theta$ естественно интерпретировать как (обобщенные) байесовские риски для моментов $\tau$ и $\bar{\tau}$, характеризующие запаздывание в обнаружении момента появления разладки, когда в роли априорного распределения для $\theta$ берется (как «наихудшее») «равномерное» распределение на $[0, \infty)$.

Отыскание величин $B(T)$ и $\bar{B}(T)$, а также соответствуюших оптимальных моментов, если они существуют, будем называть задачами о разладке в «вариантах (B) $u(\overline{\mathrm{B}}) \gg$ соответственно.

Величины $C(T)$ и $\bar{C}(T)$, определенные в $(3)$, являются минимаксными рисками. Их отыскание и отыскание соответствующих оптимальных или $\varepsilon$-оптимальных моментов остановки будем называть задачами о разладке в «вариантаx $(\mathrm{C}) u(\overline{\mathrm{C}}) \gg$.

1.4. Изложим кратко результаты, которые были получены в [11] для вариантов (В) и (C). Это даст лучшее понимание того, зачем (в вариантах $(\overline{\mathrm{B}})$ и $(\overline{\mathrm{C}}))$ приходится привлекать к рассмотрению рандомизированные моменты остановки.

Ключевую роль при решении задачи о разладке в варианте (B) играет процесс $\psi=\left(\psi_{t}\right)_{t \geqslant 0}$, построенный по наблюдаемому процессу $X=\left(X_{t}\right)_{t \geqslant 0}$ как решение стохастического дифференциального уравнения

$$
d \psi_{t}=d t+\frac{\mu}{\sigma^{2}} \psi_{t} d X_{t}, \quad \psi_{0}=0
$$

и допускающий представление

$$
\psi_{t}=\int_{0}^{t} \frac{L_{t}}{L_{\theta}} d \theta, \quad t \geqslant 0
$$

где

$$
L_{t}=\frac{d\left(\mathbf{P}_{0} \mid \mathscr{F}_{t}^{X}\right)}{d\left(\mathbf{P}_{\infty} \mid \mathscr{F}_{t}^{X}\right)}, \quad \mathscr{F}_{t}^{X}=\sigma\left(X_{s}, s \leqslant t\right)
$$

Хорошо известно (см., например, [4]), что в модели (1)

$$
L_{t}=\exp \left\{\frac{\mu}{\sigma^{2}} X_{t}-\frac{1}{2} \frac{\mu^{2}}{\sigma^{2}} t\right\} \text {. }
$$

По формуле Ито

$$
d L_{t}=\frac{\mu}{\sigma^{2}} L_{t} d X_{t}, \quad L_{0}=1
$$


То, что процесс $\left(\psi_{t}\right)_{t \geqslant 0}$, задаваемый формулой (5), является решением уравнения (4), проверяется применением формулы Ито к произведению $L_{t} \int_{0}^{t}\left(1 / L_{\theta}\right) d \theta, t \geqslant 0$.

$\mathrm{B}$ дальнейшем будем предполагать, что $\mu$ и $\sigma$ таковы, что «отношение сигнал/шум» $\rho=\mu^{2} /\left(2 \sigma^{2}\right)$ равно единице.

Это предположение упрощает приводимые далее формулы и в то же время не является ограничением, поскольку все рассматриваемые характеристики $(B(T), \bar{B}(T), \ldots)$ зависят от $\mu$ и $\sigma$ только через «отношение сигнал/шум» $\rho$ и пересчет формул от случая $\rho=1$ на общий случай произвольных значений $\rho>0$ не представляет трудностей (см. [11]).

Следующее предложение резюмирует полученные в [11] результаты в варианте (В).

Предложение 1. І. Для всякого $T>0$

(а) выполнено равенство

$$
B(T)=\inf _{\tau \in \mathfrak{M}_{T}} \frac{1}{T} \mathbf{E}_{\infty} \int_{0}^{\tau} \psi_{t} d t
$$

(b) $\inf _{\tau \in \mathfrak{M}_{T}}$ в (6) достигается на моменте

$$
\tau_{T}^{*}=\inf \left\{t \geqslant 0: \psi_{t} \geqslant T\right\}
$$

( $и$, тем самьм, этот момент является оптимальным в классе $\mathfrak{M}_{T}$ в варианте (B));

(c) выполнено равенство

$$
B(T)=F\left(T^{-1}\right)-\Delta\left(T^{-1}\right),
$$

где для всякого $b>0$

$$
F(b)=e^{b}(-\operatorname{Ei}(-b)), \quad \Delta(b)=1-b \int_{0}^{\infty} e^{-b u} \frac{\ln (1+u)}{u} d u
$$

$u-\operatorname{Ei}(-b)=\int_{b}^{\infty} t^{-1} e^{-t} d t-$ интегральная показательная функиия.

II. При мальхх и больших T

$$
B(T)= \begin{cases}\frac{T}{2}+O\left(T^{2}\right), & T \rightarrow 0, \\ \ln T-(1+\mathbb{C})+O\left(T^{-1} \ln ^{2} T\right), & T \rightarrow \infty,\end{cases}
$$

где $\mathbb{C}=0.577 \ldots-$ константа Эйлера.

1.5. Для изложения результатов в варианте (C) заметим, прежде всего, что если $\tau \in \mathfrak{M}_{T}$, то

$$
\begin{aligned}
\frac{1}{T} \int_{0}^{\infty} \mathbf{E}_{\theta}(\tau-\theta)^{+} d \theta & =\frac{1}{T} \int_{0}^{\infty} \mathbf{E}_{\theta}(\tau-\theta \mid \tau \geqslant \theta) \mathbf{P}_{\theta}\{\tau \geqslant \theta\} d \theta \\
& =\frac{1}{T} \int_{0}^{\infty} \mathbf{E}_{\theta}(\tau-\theta \mid \tau \geqslant \theta) \mathbf{P}_{\infty}\{\tau \geqslant \theta\} d \theta
\end{aligned}
$$


поскольку $\{\tau \geqslant \theta\} \in \mathscr{F}_{\theta}^{X}$, а для событий $A \in \mathscr{F}_{\theta}^{X}$ и любого $\theta>0$ вероятность $\mathbf{P}_{\theta}(A)$ та же самая, что и вероятность $\mathbf{P}_{\infty}(A)$.

Из (10) и равенств $T^{-1} \int_{0}^{\infty} \mathbf{P}_{\infty}\{\tau \geqslant \theta\} d \theta=T^{-1} \mathbf{E}_{\infty} \tau=1$, получаем

$$
\begin{aligned}
\frac{1}{T} \int_{0}^{\infty} \mathbf{E}_{\theta}(\tau-\theta)^{+} d \theta & \leqslant \frac{1}{T} \int_{0}^{\infty}\left\{\sup _{\theta \geqslant 0} \mathbf{E}_{\theta}(\tau-\theta \mid \tau \geqslant \theta)\right\} \mathbf{P}_{\infty}(\tau \geqslant \theta) d \theta \\
& =\sup _{\theta \geqslant 0} \mathbf{E}_{\theta}(\tau-\theta \mid \tau \geqslant \theta) .
\end{aligned}
$$

Следовательно, $\inf _{\tau \in \mathfrak{M}_{T}} T^{-1} \int_{0}^{\infty} \mathbf{E}_{\theta}(\tau-\theta)^{+} d \theta \leqslant \inf _{\tau \in \mathfrak{M}_{T}} \sup _{\theta} \mathbf{E}_{\theta}(\tau-$ $\theta \mid \tau \geqslant \theta)$, т.e.

$$
B(T) \leqslant C(T),
$$

что и дает для величины $C(T)$ оценку снизу. Объединяя эту оценку и оценку сверху $C(T) \leqslant F\left(T^{-1}\right)\left(=C^{*}(T)\right)$, полученную в [11], можно сформулировать следующий результат.

Предложение 2. В варианте (C) для величинь $C(T)$ справедливы следуюшие неравенства:

$$
F\left(T^{-1}\right)-\Delta\left(T^{-1}\right) \leqslant C(T) \leqslant F\left(T^{-1}\right) .
$$

При этом для мальх $T$

$$
\frac{T}{2}+O\left(T^{2}\right) \leqslant C(T) \leqslant T+O\left(T^{2}\right)
$$

а для больщuх $T$

$$
\ln T-(1+\mathbb{C})+O\left(T^{-1} \ln ^{2} T\right) \leqslant C(T) \leqslant \ln T-\mathbb{C}+O\left(T^{-1} \ln ^{2} T\right) .
$$

Из формулы (13) видно, что при больших $T$ (а именно этот случай представляет основной интерес в задачах наискорейшего обнаружения) минимаксный риск $C(T)$ ведет себя как $\ln T$ (в том смысле, что $C(T) / \ln T \rightarrow 1, T \rightarrow \infty ;$ асимптотика первого порядка), в то же время вторые члены (а именно, $-(1+\mathbb{C})$ и $-\mathbb{C})$ отличаются на единицу. Следующие же члены стремятся к нулю как $O\left(T^{-1} \ln ^{2} T\right), T \rightarrow \infty$.

1.6. Основная цель настоящей работы состоит в том, чтобы ликвидировать «зазор» во вторых членах в левой и правой частях неравенств (13). Это оказывается возможным, если вместо $C(T)$ рассматривать меньшую величину $\bar{C}(T)$, являюшуюся минимаксным риском (см. (3)) по классу $\overline{\mathfrak{M}}_{T}$ рандомизированных моментов остановки. (Отметим, что в задачах скорейшего обнаружения для случая дискретного времени рандомизированные моменты остановки рассматривались в [20].) Как следует из приводимых далее результатов, при больших $T$ выполнены неравенства

$$
\ln T-(1+\mathbb{C})+O\left(T^{-1} \ln ^{2} T\right) \leqslant \bar{C}(T) \leqslant \ln T-(1+\mathbb{C})+O\left(T^{-1} \ln ^{2} T\right) .
$$


Из (14) видно, что для $\bar{C}(T)$ имеет место асимптотика второго порядка

$$
\bar{C}(T)=\ln T-(1+\mathbb{C})+O\left(T^{-1} \ln ^{2} T\right), \quad T \rightarrow \infty,
$$

из которой, конечно, следует (более грубая) асимптотика

$$
\frac{\bar{C}(T)}{\ln T-(1+\mathbb{C})} \rightarrow 1, \quad T \rightarrow \infty \text {. }
$$

\section{2. О методе получения оценок сверху и снизу для $\bar{C}(T)$}

2.1. По аналогии с (12) получаем неравенство $\bar{B}(T) \leqslant \bar{C}(T)$.

Для $B(T)$ справедливо представление (4), и аналогичное представление (тем же самым методом, что и в [11]) получаем для $\bar{B}(T)$ :

$$
\bar{B}(T)=\inf _{\bar{\tau} \in \overline{\mathfrak{M}}_{T}} \frac{1}{T} \overline{\mathbf{E}}_{\infty} \int_{0}^{\bar{\tau}} \psi_{t}(\bar{\omega}) d t \quad \text { при всех } \quad T>0,
$$

где $\psi_{t}(\bar{\omega})=\psi_{t}(\omega)$ для $\bar{\omega}=(\omega, \widetilde{\omega})$.

Относительно меры $\mathbf{P}_{\infty}$ процесс $\psi=\left(\psi_{t}(\omega)\right)_{t \geqslant 0}$ является диффузионным марковским процессом с дифференциалом

$$
d \psi_{t}(\omega)=d t+\frac{\mu}{\sigma} \psi_{t}(\omega) d B_{t}(\omega), \quad \psi_{0}(\omega)=0
$$

Из общей теории оптимальных правил остановки для марковских процессов следует, что

$$
\bar{B}(T)=B(T)
$$

Иначе говоря, взятие инфимума в (6) не по классу $\mathfrak{M}_{T}$, а по более широкому классу $\overline{\mathfrak{M}}_{T}$ рандомизированных моментов ничего нового не дает. (По поводу того, что в марковских задачах об оптимальной остановке $\inf _{\tau \in \mathfrak{M}_{T}}$ совпадает с $\inf _{\bar{\tau} \in \overline{\mathfrak{M}}_{T}}$, см. [8, гл. II, $\S 7$, с. 100].) С учетом приведенного неравенства $\bar{B}(T) \leqslant \bar{C}(T)$ и формулы $(7)$, из (15) получаем нижнюю оценку для $\bar{C}(T)$ : при всех $T>0$

$$
F\left(T^{-1}\right)-\Delta\left(T^{-1}\right) \leqslant \bar{C}(T) .
$$

2.2. Чтобы получить верхнюю оценку для $\bar{C}(T)$, воспользуемся следующими соображениями.

Ясно, что для конкретного момента $\bar{\tau}^{*}$ из $\overline{\mathfrak{M}}_{T}$ справедливо неравенство

$$
\bar{C}(T) \leqslant \sup _{\theta \geqslant 0} \overline{\mathbf{E}}_{\theta}\left(\bar{\tau}^{*}-\theta \mid \bar{\tau}^{*} \geqslant \theta\right) .
$$

Тем самым, имея в виду отмеченное в п. 1.1 желание уменьшить «зазор» между верхней и нижней оценками, надо подобрать конкретный момент $\bar{\tau}^{*} \in \overline{\mathfrak{M}}_{T}$ так, чтобы значение $\sup _{\theta} \overline{\mathbf{E}}_{\theta}\left(\bar{\tau}^{*}-\theta \mid \bar{\tau}^{*} \geqslant \theta\right)$ было как 
можно ближе к значению $\bar{B}(T)(=B(T))$. Как станет ясно из дальнейшего, приводимый ниже момент $\bar{\tau}_{g_{A}}^{*}$ из класса $\overline{\mathfrak{M}}_{T}$ со специально подобранным значением $A=A(T)$, «почти» удовлетворяет желаемому свойству уменьшения «зазора», по крайней мере для больших значений $T$.

Момент $\bar{\tau}_{g_{A}}^{*}$ строится следуюшим образом. Пусть $\psi^{*}=\left(\psi_{t}^{*}(\bar{\omega})\right)_{t \geqslant 0}-$ процесс со стохастическим дифференциалом

$$
d \psi_{t}^{*}(\bar{\omega})=d t+\frac{\mu}{\sigma^{2}} \psi_{t}^{*}(\bar{\omega}) d X_{t}(\bar{\omega})
$$

$\mathbb{L}_{\infty}$ - сопряженный инфинитезимальный оператор (см. [4]) процесса $\psi=\left(\psi_{t}\right)_{t \geqslant 0} \mathrm{c}$ дифференциалом $d \psi_{t}=d t+(\mu / \sigma) \psi_{t} d B_{t}, \psi_{0}=0$ $\left(\rho \equiv \mu^{2} /\left(2 \sigma^{2}\right)=1\right)$. Очевидно, что $\mathbb{L}_{\infty}=\partial^{2}\left(y^{2} \cdot\right) / \partial y^{2}-\partial / \partial y$. Обозначим через $g_{A}(y), 0 \leqslant y \leqslant A$, плотность распределения вероятностей, удовлетворяющую при $0 \leqslant y<A$ уравнению

$$
\mathbb{L}_{\infty} g_{A}=0,
$$

граничному условию $g_{A}(A)=0$ и условию нормировки $\int_{0}^{A} g_{A}(y) d y=1$.

Возьмем начальное значение $\psi_{0}^{*}(\bar{\omega})$ процесса $\psi^{*}=\left(\psi_{t}^{*}(\bar{\omega})\right)_{t \geqslant 0}$ случайным, не зависящим от броуновского движения $B(\bar{\omega})=\left(B_{t}(\omega, \widetilde{\omega})\right)_{t \geqslant 0}$ $\left(B_{t}(\omega, \widetilde{\omega})=B_{t}(\omega), t \geqslant 0\right)$ и с таким распределением вероятностей $G_{A}(y)=\overline{\mathbf{P}}\left\{\psi_{0}^{*}(\bar{\omega}) \leqslant y\right\}, y \in[0, A]$, что $G_{A}(y)=\int_{0}^{y} g_{A}(x) d x$. Положим

$$
\bar{\tau}_{g_{A}}^{*}=\inf \left\{t \geqslant 0: \psi_{t}^{*}(\bar{\omega}) \geqslant A\right\} .
$$

В силу неравенства (17) и предполагаемой независимости начального значения $\psi_{0}^{*}(\bar{\omega})$ от броуновского движения $B=\left(B_{t}(\bar{\omega})\right)_{t \geqslant 0}$ получаем, что для момента $\bar{\tau}_{g_{A}}^{*}$ выполнены неравенства

$$
\begin{aligned}
\bar{C}(T) & \leqslant \sup _{\theta} \overline{\mathbf{E}}_{\theta}\left(\bar{\tau}_{g_{A}}^{*}-\theta \mid \bar{\tau}_{g_{A}}^{*} \geqslant \theta\right) \\
& =\sup _{\theta} \int_{0}^{A} \mathbf{E}_{\theta}\left(\tau_{A}^{(y)}(\omega)-\theta \mid \tau_{A}^{(y)}(\omega) \geqslant \theta\right) g_{A}(y) d y \\
& \leqslant \int_{0}^{A} \sup _{\theta}\left\{\mathbf{E}_{\theta}\left(\tau_{A}^{(y)}(\omega)-\theta \mid \tau_{A}^{(y)}(\omega) \geqslant \theta\right)\right\} g_{A}(y) d y \\
& =\int_{0}^{A} \mathbf{E}_{0} \tau_{A}^{(y)}(\omega) g_{A}(y) d y=\overline{\mathbf{E}}_{0} \bar{\tau}_{g_{A}}^{*},
\end{aligned}
$$

где

$$
\tau_{A}^{(y)}(\omega)=\inf \left\{t \geqslant 0: \psi_{t}^{(y)}(\omega) \geqslant A\right\}, \quad d \psi_{t}^{(y)}(\omega)=d t+\frac{\mu}{\sigma^{2}} \psi_{t}^{(y)}(\omega) d X_{t}(\omega)
$$

с $\psi_{0}^{(y)}=y, 0 \leqslant y \leqslant A$ (первое равенство в $(21)$ следует из доказательства теоремы 3.1 статьи [11]).

Тем самым, для $\bar{C}(T)$ при всех $T>0$ имеем следующие оценки сверху и снизу:

$$
F\left(T^{-1}\right)-\Delta\left(T^{-1}\right) \leqslant \bar{C}(T) \leqslant \overline{\mathbf{E}}_{0} \bar{\tau}_{g_{A}}^{*},
$$


где порог $A=A(T)$ должен выбираться из тех соображений, что $\bar{\tau}_{g_{A}}^{*} \in \overline{\mathfrak{M}}_{T}$, т.е. так, что $\overline{\mathbf{E}}_{\infty} \bar{\tau}_{g_{A}}^{*}=T$.

Итак,

$$
\overline{\mathbf{E}}_{0} \bar{\tau}_{g_{A}}^{*}=\int_{0}^{A} \mathbf{E}_{0} \tau_{A}^{(y)}(\omega) g_{A}(y) d y, \quad \overline{\mathbf{E}}_{\infty} \bar{\tau}_{g_{A}}^{*}=\int_{0}^{A} \mathbf{E}_{\infty} \tau_{A}^{(y)}(\omega) g_{A}(y) d y .
$$

Таким образом, для отыскания верхней границы $\overline{\mathbf{E}}_{0} \bar{\tau}_{g_{A}}^{*}$ в $(22)$ необходимо прежде всего найти рандомизирующее распределение $G_{A}=G_{A}(y), 0 \leqslant$ $y \leqslant A$, с плотностью $g_{A}(y)$ и затем найти $\overline{\mathbf{E}}_{\infty} \bar{\tau}_{g_{A}}^{*}$, что и будет сделано в разделах 3 и 4.

\section{3. Нахождение рандомизирующей плотности $g_{A}$}

3.1. Плотность $g_{A}=g_{A}(y), 0 \leqslant y \leqslant A$, ищется как неотрицательное решение уравнения (19), подчиняющееся граничному условию $g_{A}(A)=0$ и условию нормировки $\int_{0}^{A} g_{A}(y) d y=1$. В следующей лемме дается явная формула для этой плотности.

Лемма 1. Плотность $g_{A}=g_{A}(y), 0 \leqslant y \leqslant A$, задается формулой

$$
g_{A}(y)=f(y) \frac{h(A)-h(y)}{A}
$$

$2 \partial e$

$$
f(y)=y^{-2} e^{1 / y}, \quad h(y)=y e^{1 / y}-\operatorname{Ei}\left(y^{-1}\right),
$$

и интегральная показательная функиия - $\mathrm{Ei}(z)$ определяется следующим образом (см. [2]):

$$
-\operatorname{Ei}(z)= \begin{cases}\int_{-z}^{\infty} \frac{e^{-t}}{t} d t, & z<0, \\ \lim _{\varepsilon \rightarrow+0}\left[\int_{-z}^{-\varepsilon} \frac{e^{-t}}{t} d t+\int_{\varepsilon}^{\infty} \frac{e^{-t}}{t} d t\right], & z>0 .\end{cases}
$$

(Если $z>0$, то $-\mathrm{Ei}(-z)=\int_{z}^{\infty} t^{-1} e^{-t} d t$, что уже встречалось в предложении 1.)

Д ок азате ль с т в о. Зафиксируем $A>0$ и вместо $g_{A}$ будем писать $g$. Производя в (19) интегрирование, находим, что $g=\left(y^{2} g\right)^{\prime}+C_{1}$, откуда

$$
g^{\prime}=g\left(y^{-2}-2 y^{-1}\right)-C_{1} y^{-2} .
$$

Будем искать решение этого уравнения в виде

$$
g(y)=f(y) u(y)
$$

где $f(y)=y^{-2} e^{-1 / y}$. Тогда

$$
g^{\prime}(y)=(f(y) u(y))^{\prime}=f(y) u(y)\left(y^{-2}-2 y^{-1}\right)+f(y) u^{\prime}(y) .
$$


Из (27) и (28) получаем, что

$$
g^{\prime}(y)=f(y) u(y)\left(y^{-2}-2 y^{-1}\right)-C_{1} y^{-2}
$$

Сравнивая это выражение с (29), находим, что $f(y) u^{\prime}(y)=-C_{1} / y^{2}$. Отсюда $u^{\prime}(y)=-C_{1} e^{1 / y}$ и, значит,

$$
\begin{aligned}
u(y) & =C_{2}-C_{1} \int_{0}^{y} e^{1 / z} d z=C_{2}+C_{1} \int_{\infty}^{1 / y} \frac{e^{t}}{t^{2}} d t=C_{2}-C_{1} \int_{1 / y}^{\infty} \frac{e^{t}}{t^{2}} d t \\
& =C_{2}+C_{1}\left[-y e^{1 / y}+\operatorname{Ei}\left(y^{-1}\right)\right] .
\end{aligned}
$$

Тем самым для $g(y)=f(y) u(y)$ получаем, что

$$
g(y)=C_{1}\left[-y^{-1}+f(y) \operatorname{Ei}\left(y^{-1}\right)\right]+C_{2} f(y)
$$

Определим константы $C_{1}$ и $C_{2}$ из условий $g(A)=0$ и $\int_{0}^{A} g(y) d y=1$. С этой целью обозначим

$$
I_{1}=\int_{0}^{A}\left[-y^{-1}+f(y) \operatorname{Ei}\left(y^{-1}\right)\right] d y, \quad I_{2}=\int_{0}^{A} f(y) d y
$$

Тогда условие $\int_{0}^{A} g(y) d y=1$ примет вид $C_{1} I_{1}+C_{2} I_{2}=1$. Найдем $I_{1}$ и $I_{2}$. Имеем

$$
I_{1}=\int_{0}^{A}\left[-y^{-1}+y^{-2} \operatorname{Ei}\left(y^{-1}\right) e^{-1 / y}\right] d y .
$$

Известно (см. 8.212.2 в [2]), что $\operatorname{Ei}(z)=e^{z}\left(z^{-1}+\int_{0}^{\infty}(z-t)^{-2} e^{-t} d t\right)$ для $z>0$. Значит,

$$
y^{-2} \operatorname{Ei}\left(y^{-1}\right) e^{-1 / y}=y^{-1}+y^{-2} \int_{0}^{\infty} \frac{e^{-t} d t}{(1 / y-t)^{2}}
$$

и из (32) находим, что

$$
\begin{aligned}
I_{1} & =\int_{0}^{A} \frac{1}{y^{2}}\left[\int_{0}^{\infty} \frac{e^{-t}}{(1 / y-t)^{2}} d t\right] d y=\int_{0}^{\infty} e^{-t}\left[\int_{0}^{A} \frac{d y}{(1-y t)^{2}}\right] d t \\
& =\int_{0}^{\infty} \frac{e^{-t}}{t^{2}}\left[\int_{0}^{A} \frac{d y}{(1 / t-y)^{2}}\right] d t=\int_{0}^{\infty} \frac{e^{-t}}{t^{2}}\left(\frac{1}{1 / t-A}-\frac{1}{1 / t}\right) d t \\
& =\int_{0}^{\infty} \frac{e^{-t}}{1 / A-t} d t .
\end{aligned}
$$

Воспользовавшись здесь известной формулой

$$
\operatorname{Ei}( \pm x y)= \pm e^{ \pm x y} \int_{0}^{\infty} \frac{e^{-x t}}{y \mp t} d t, \quad \text { Re } y>0, \quad x>0
$$

(см. $[2,8.212 .5])$, получаем, что $I_{1}=e^{-1 / A} \operatorname{Ei}\left(A^{-1}\right)$. 
Для $I_{2}$ имеем:

$$
I_{2}=\int_{0}^{A} f(y) d y=\int_{0}^{A} y^{-2} e^{-1 / y} d y=e^{-1 / A} .
$$

Тем самым, условие $C_{1} I_{1}+C_{2} I_{2}=1$ (т.е. условие $\int_{0}^{A} g(y) d y=1$ ) принимает вид $C_{1} e^{-1 / A} \operatorname{Ei}\left(A^{-1}\right)+C_{2} e^{-1 / A}=1$, а условие $g(A)=0$ превращается в условие $C_{1}\left(-A e^{1 / A}+\operatorname{Ei}\left(A^{-1}\right)\right)+C_{2}=0$.

Из этих двух равенств находим, что $C_{1}=A^{-1}, C_{2}=e^{1 / A}-$ $\operatorname{Ei}\left(A^{-1}\right) A^{-1}$, и формула (31) преобразуется к требуемому виду (24). Лемма 1 доказана.

3.2. Обозначим через $Q_{A}(y)$ такую вероятностную меру на множестве $[0, A]$, что для процесса $\psi^{*}=\left(\psi_{t}^{*}(\bar{\omega})\right)_{t \geqslant 0}$ со стохастическим дифференциалом $(18)$ и случайным начальным значением $\psi_{0}^{*}(\bar{\omega})$, не зависящим от броуновского движения $B(\bar{\omega})=\left(B_{t}(\omega, \widetilde{\omega})\right)_{t \geqslant 0}\left(\right.$ где $B_{t}(\omega, \widetilde{\omega})=B_{t}(\omega)$, $t \geqslant 0)$ и распределенным по мере $Q_{A}(y)$ (т.е. $\overline{\mathbf{P}}\left\{\psi_{0}^{*}(\bar{\omega}) \leqslant y\right\}=Q_{A}(y)$, $y \in[0, A])$, при всех $t>0$ выполняется равенство

$$
\overline{\mathbf{P}}_{\infty}\left(\psi_{t}^{*}(\bar{\omega}) \leqslant y \mid \psi_{s}^{*}(\bar{\omega})<A, s \leqslant t\right)=Q_{A}(y) .
$$

Как известно (см., например, [9], [10], [13]-[18], [23]), распределение $Q_{A}(y), y \in[0, A]$, для начального состояния $\psi_{0}^{*}(\bar{\omega})$ со свойством (34) принято называть квазистаиионарным.

Согласно результатам статьи [9] (см. также [10], [14], [17], [23]), квазистационарное распределение $Q_{A}(y)$ имеет плотность $q_{A}(y)$, которая удовлетворяет уравнению

$$
\mathbb{L}_{\infty} q_{A}(y)=-\lambda_{1} q_{A}(y), \quad y \in[0, A],
$$

где $\mathbb{L}_{\infty}=\partial^{2}\left(y^{2} \cdot\right) / \partial y^{2}-\partial / \partial y-$ сопряженный инфинитезимальный оператор процесса $\psi=\left(\psi_{t}\right)_{t \geqslant 0}$ с дифференциалом $d \psi_{t}=d t+(\mu / \sigma) \psi_{t} d B_{t}$, $\psi_{0}=0\left(\rho \equiv \mu^{2} /\left(2 \sigma^{2}\right)=1\right)$, а $\lambda_{1}=\lambda_{1}(A)>0$ - наименьшее собственное число оператора $\mathbb{L}_{\infty}$ (в рассматриваемом случае этот оператор будет иметь чисто дискретный спектр). При этом плотность $q_{A}(y)$ должна удовлетворять граничным условиям $q_{A}(A)=0, q_{A}^{\prime}(A)=-A^{-2} e^{1-1 / A}$ и условию нормировки $\int_{0}^{A} q_{A}(y) d y=1$.

Согласно результатам работ [9], [18],

$$
\lim _{A \rightarrow \infty} Q_{A}(y)=Q_{\infty}(y), \quad y>0,
$$

где $Q_{\infty}(y)=\lim _{t \rightarrow \infty} \mathbf{P}_{\infty}\left\{\psi_{t} \leqslant y\right\}, y>0$, является стаиионарньцм (инвариантным) распределением процесса $\psi=\left(\psi_{t}\right)_{t \geqslant 0}$ (определение процесса см. выше). При этом стационарное распределение $Q_{\infty}(y)$ также имеет плотность $q_{\infty}(y)$, которая удовлетворяет уравнению

$$
\mathbb{L}_{\infty} q_{\infty}(y)=0, \quad y>0,
$$


с граничным условием $\lim _{y \rightarrow \infty}\left\{\left(y^{2} q_{\infty}(y)\right)^{\prime}-q_{\infty}(y)\right\}=0$, а также условию нормировки $\int_{0}^{\infty} q_{\infty}(y) d y=1$ (см. также [11], [19]). Таким образом, из формул (35)-(37) следует, что $\lim _{A \rightarrow \infty} \lambda_{1}(A)=0$. Поэтому при $A \rightarrow \infty$ решение $q_{A}(y)$ уравнения (35) можно разложить по степеням $\lambda_{1}(A)$ как по малому параметру, т.е. представить $q_{A}(y)$ в виде ряда $q_{A}(y)=q_{A}^{0}(y)+$ $\lambda_{1}(A) q_{A}^{1}(y)+\cdots, y \in[0, A]$, где функции $q_{A}^{0}(y)$ и $q_{A}^{1}(y)$ удовлетворяют уравнениям

$$
\mathbb{L}_{\infty} q_{A}^{0}(y)=0, \quad \mathbb{L}_{\infty} q_{A}^{1}(y)=-q_{A}^{0}(y), \quad y \in[0, A],
$$

граничным условиям $q_{A}^{0}(A)=0$ и $q_{A}^{1}(A)=0$, условиям нормировки $\int_{0}^{A} q_{A}^{0}(y) d y=1$ и $\int_{0}^{A} q_{A}^{1}(y) d y=0$ соответственно. Таким образом, плотность $g_{A}(y)\left(\equiv q_{A}^{0}(y)\right)$ рандомизирующего распределения $G_{A}(y)$ является ничем иным, как нулевым членом в разложении плотности квазистационарного распределения $Q_{A}(y)$ по степеням собственного числа $\lambda_{1}(A) \rightarrow 0$ сопряженного инфинитезимального оператора $\mathbb{L}_{\infty}$ процесса $\psi=\left(\psi_{t}\right)_{t \geqslant 0}$ при $A \rightarrow \infty$.

Заметим, что из разложения $q_{A}(y)$ по степеням $\lambda_{1}(A)$ при $A \rightarrow \infty$ и условия $q_{A}^{\prime}(A)=-A^{-2} e^{1-1 / A}$ следует асимптотика

$$
\lambda_{1}(A)=6 e A^{-1}+O\left(A^{-2}\right), \quad A \rightarrow \infty .
$$

3 а м е ч а н и е 1. Отметим, что если бы для момента $\bar{\tau}_{g_{A}}^{*}$ из $(20)$ начальное значение процесса $\psi^{*}=\left(\psi_{t}^{*}(\bar{\omega})\right)_{t \geqslant 0}$ рандомизировалось не с помощью распределения $G_{A}(y)$, а с помощью квазистационарного распределения $Q_{A}(y)$, то в силу (34) было бы выполнено равенство (ср. с (21))

$$
\sup _{\theta} \overline{\mathbf{E}}_{\theta}\left(\bar{\tau}_{g_{A}}^{*}-\theta \mid \bar{\tau}_{g_{A}}^{*} \geqslant \theta\right)=\overline{\mathbf{E}}_{0} \bar{\tau}_{g_{A}}^{*}, \quad \theta \geqslant 0 .
$$

3 а м е ч а н и е 2. Несложно подсчитать, что $q_{\infty}(y)=y^{-2} e^{-1 / y}$, $y>0$, т.е. для предельного распределения $Q_{\infty}(y)=\int_{0}^{y} q_{\infty}(x) d x$ получаем формулу

$$
Q_{\infty}(y)=e^{-1 / y}, \quad y>0 .
$$

В статистике экстремальных значений это распределение известно под названием «распределение типа Фреше» (см. [12]).

Найденные величины $C_{1}=C_{1}(A)$ и $C_{2}=C_{2}(A)$ таковы, что $C_{1}(A) \rightarrow 0, C_{2}(A) \rightarrow 1$ при $A \rightarrow \infty$. С учетом этого из $(24)$ находим, $\lim _{A \rightarrow \infty} g_{A}(y)=q_{\infty}(y)$, как и следовало ожидать.

\section{4. Вычисление $\overline{\mathrm{E}}_{\infty} \bar{\tau}_{g_{A}}^{*}$}

4.1. Согласно второму равенству в $(23)$, для вычисления $\overline{\mathbf{E}} \bar{\tau}_{g_{A}}^{*}$ прежде всего надо найти $\mathbf{E}_{\infty} \tau_{A}^{(y)}$, где для $0 \leqslant y \leqslant A$

$$
\tau_{A}^{(y)}=\inf \left\{t \geqslant 0: \psi_{t}^{(y)} \geqslant A\right\}
$$


с процессом $\left(\psi_{t}^{(y)}\right)_{t \geqslant 0}$, для которого

$$
\psi_{t}^{(y)}=y+t+\frac{\mu}{\sigma} \int_{0}^{t} \psi_{s}^{(y)} d B_{s}, \quad t \geqslant 0 .
$$

Поскольку $\psi_{t \wedge \tau_{A}^{(y)}}^{(y)} \leqslant A$ и процесс $\left(\int_{0}^{t} \psi_{s}^{(y)} d B_{s}\right)_{t \geqslant 0}$ является локальным мартингалом, то $\mathbf{E}_{\infty} \int_{0}^{t \wedge \tau_{A}^{(y)}} \psi_{s}^{(y)} d B_{s}=0$, и из $(38)$ находим, что $y+\mathbf{E}_{\infty}(t \wedge$ $\left.\tau_{A}^{(y)}\right) \leqslant A$ для всякого $t>0$ и, значит, $\mathbf{E}_{\infty}\left(t \wedge \tau_{A}^{(y)}\right) \leqslant A-y$, откуда предельным переходом по $t \rightarrow \infty$ получаем (по теореме о монотонной сходимости), что

$$
\mathbf{E}_{\infty} \tau_{A}^{(y)} \leqslant A-y<\infty .
$$

Из (38) и (39), применяя «первое тождество Вальда» (см. [5]) к мартингалу $\left(\int_{0}^{t \wedge \tau_{A}^{(y)}} \psi_{s}^{(y)} d B_{s}\right)_{t \geqslant 0}$, находим, что

$$
\mathbf{E}_{\infty} \psi_{\tau_{A}^{(y)}}^{(y)}=y+\mathbf{E}_{\infty} \tau_{A}^{(y)}
$$

Из теоремы сравнения для стохастических дифференциальных уравнений (см. [1]) следует, что $\psi_{t}^{(y)} \geqslant L_{t}^{(y)}$ (P-п.н.), $t \geqslant 0$, где $L_{t}^{(y)}$ имеет дифференциал

$$
d L_{t}^{(y)}=\frac{\mu}{\sigma} L_{t}^{(y)} d B_{t}, \quad L_{0}^{(y)}=y
$$

Поскольку решение уравнения (41) имеет вид

$$
L_{t}^{(y)}=y \exp \left\{\frac{\mu}{\sigma} B_{t}-\frac{1}{2} \frac{\mu^{2}}{\sigma^{2}} t\right\}
$$

из которого следует, что при $y>0$ процесс $L_{t}^{(y)}$ с вероятностью единица выходит на любой уровень $A>0$, то заключаем, что и процесс $\psi_{t}^{(y)}$, $t \geqslant 0$, с вероятностью единица выходит на любой уровень $A>0$. Тем самым, $\psi_{\tau_{A}^{(y)}}^{(y)}=A$ (P-п.н.) и из (40) получаем, что $\mathbf{E}_{\infty} \tau_{A}^{(y)}=A-y$ для всякого $y>0$. (По непрерывности эта формула верна и при $y=0$.)

Итак,

$$
\overline{\mathbf{E}}_{\infty} \bar{\tau}_{g_{A}}^{*}=\int_{0}^{A} \mathbf{E}_{\infty} \tau_{A}^{(y)} d G_{A}(y)=A-\int_{0}^{A} y g_{A}(y) d y
$$

4.2. Согласно (24), (25),

$$
g_{A}(y)=\frac{1}{y^{2}} e^{-1 / y} \frac{\left(A e^{1 / A}-\operatorname{Ei}(1 / A)\right)-\left(y e^{1 / y}-\operatorname{Ei}(1 / y)\right)}{A} .
$$

Значит,

$$
\begin{aligned}
\int_{0}^{A} y g_{A}(y) d y= & \left(e^{-1 / A}-\frac{1}{A} \operatorname{Ei}\left(\frac{1}{A}\right)\right) \int_{0}^{A} \frac{1}{y} e^{-1 / y} d y \\
& +\frac{1}{A} \int_{0}^{A}\left(-1+\frac{1}{y} \operatorname{Ei}\left(\frac{1}{y}\right) e^{-1 / y}\right) d y
\end{aligned}
$$


Имеем

$$
J_{1} \equiv \int_{0}^{A} \frac{1}{y} e^{-1 / y} d y=\int_{1 / A}^{\infty} \frac{e^{-z}}{z} d z=-\operatorname{Ei}\left(-\frac{1}{A}\right) .
$$

Покажем, что

$$
\begin{aligned}
J_{2} \equiv & \int_{0}^{A} \frac{1}{y} \operatorname{Ei}\left(\frac{1}{y}\right) e^{-1 / y} d y=-\operatorname{Ei}\left(-\frac{1}{A}\right) \operatorname{Ei}\left(\frac{1}{A}\right) \\
& +\int_{0}^{\infty} e^{-z / A} \frac{\ln (1+z)}{z} d z
\end{aligned}
$$

Действительно,

$$
\begin{aligned}
J_{2} & =\int_{0}^{A} \frac{1}{y} \operatorname{Ei}\left(\frac{1}{y}\right) e^{-1 / y} d y=\int_{1 / A}^{\infty} \frac{e^{-z}}{z} \operatorname{Ei}(z) d z \\
& =\int_{1 / A}^{\infty} \operatorname{Ei}(z) d(\operatorname{Ei}(-z))=\left.\operatorname{Ei}(-z) \operatorname{Ei}(z)\right|_{1 / A} ^{\infty}-\int_{1 / A}^{\infty} \operatorname{Ei}(-z) \frac{e^{z}}{z} d z
\end{aligned}
$$

По правилу Лопиталя (с учетом соотношений $\lim _{z \rightarrow \infty} \operatorname{Ei}(-z)=0$, $\lim _{z \rightarrow \infty} \operatorname{Ei}(z)=\infty$ и $\left.(\operatorname{Ei}(-z))^{\prime}=e^{-z} / z,(\operatorname{Ei}(z))^{\prime}=e^{z} / z, z>0\right)$ находим, что

$$
\begin{aligned}
& \lim _{z \rightarrow \infty} \operatorname{Ei}(-z) \operatorname{Ei}(z)=\lim _{z \rightarrow \infty} \frac{\operatorname{Ei}(z)}{(\operatorname{Ei}(-z))^{-1}}=\lim _{z \rightarrow \infty} \frac{-e^{z} / z}{\left(e^{-z} / z\right)(\operatorname{Ei}(-z))^{-2}} \\
& =-\lim _{z \rightarrow \infty} e^{2 z}(\operatorname{Ei}(-z))^{2}=-\lim _{z \rightarrow \infty}\left(-z^{-1}+O\left(z^{-2}\right)\right)^{2}=0
\end{aligned}
$$

где мы воспользовались формулой 3.2 .4 из справочника [3], согласно которой $\lim _{z \rightarrow \infty} e^{z} \operatorname{Ei}(-z)=\lim _{z \rightarrow \infty}\left(-z^{-1}+O\left(z^{-2}\right)\right)=0$.

Итак,

$$
J_{2}=-\operatorname{Ei}\left(-\frac{1}{A}\right) \operatorname{Ei}\left(\frac{1}{A}\right)+\int_{1 / A}^{\infty}(-\operatorname{Ei}(-z)) \frac{e^{z}}{z} d z
$$

Далее, по формуле (33) находим

$$
\int_{1 / A}^{\infty}(-\operatorname{Ei}(-z)) \frac{e^{z}}{z} d z=\int_{1 / A}^{\infty} e^{-z}\left(\int_{0}^{\infty} \frac{e^{-t}}{z+t} d t\right) \frac{e^{z}}{z} d z=\int_{0}^{\infty} e^{-t} K(t) d t
$$

где $K(t)=\int_{1 / A}^{\infty}[z(z+t)]^{-1} d z=t^{-1} \ln (1+A t)$.

Таким образом,

$$
\begin{aligned}
J_{2} & =-\operatorname{Ei}\left(-\frac{1}{A}\right) \operatorname{Ei}\left(\frac{1}{A}\right)+\int_{0}^{\infty} \frac{e^{-t}}{t} \ln (1+A t) d t \\
& =-\operatorname{Ei}\left(-\frac{1}{A}\right) \operatorname{Ei}\left(\frac{1}{A}\right)+\int_{0}^{\infty} e^{-z / A} \frac{\ln (1+z)}{z} d z
\end{aligned}
$$

что и дает требуемую формулу (44). 
Из (42)-(44) заключаем, что

$$
\begin{aligned}
\int_{0}^{A} y g_{A}(y) d y= & \left(e^{1 / A}-A^{-1} \operatorname{Ei}\left(A^{-1}\right)\right) J_{1}-1+A^{-1} J_{2} \\
= & -1-\left(e^{1 / A}-A^{-1} \operatorname{Ei}\left(A^{-1}\right)\right) \operatorname{Ei}\left(-A^{-1}\right) \\
& +A^{-1}\left(-\operatorname{Ei}\left(-A^{-1}\right) \operatorname{Ei}\left(A^{-1}\right)+\int_{0}^{\infty} e^{-z / A} \frac{\ln (1+z)}{z} d z\right) \\
= & e^{1 / A}\left(-\operatorname{Ei}\left(-A^{-1}\right)\right)-\left(1-A^{-1} \int_{0}^{\infty} e^{-z / A} \frac{\ln (1+z)}{z} d z\right) \\
= & F\left(A^{-1}\right)-\Delta\left(A^{-1}\right),
\end{aligned}
$$

где $F(b)$ и $\Delta(b)$ определены в (8). В [11] показано, что

$$
F\left(A^{-1}\right)=e^{1 / A}\left(-\operatorname{Ei}\left(-A^{-1}\right)\right)= \begin{cases}A+O\left(A^{2}\right), & A \rightarrow 0 \\ \ln A-\mathbb{C}+O\left(A^{-1} \ln A\right), & A \rightarrow \infty\end{cases}
$$

где $\mathbb{C}=0.577 \ldots$ - константа Эйлера, и

$$
\begin{aligned}
\Delta\left(A^{-1}\right) & =1-A^{-1} \int_{0}^{\infty} e^{-z / A} \frac{\ln (1+z)}{z} d z \\
& = \begin{cases}\frac{A}{2}+O\left(A^{2}\right), & A \rightarrow 0, \\
1+O\left(A^{-1} \ln ^{2} A\right), & A \rightarrow \infty .\end{cases}
\end{aligned}
$$

Итак, доказана следующая лемма.

Лемма 2. Для любого $A>0$

$$
\overline{\mathbf{E}}_{\infty} \bar{\tau}_{g_{A}}^{*}=A-\left[F\left(A^{-1}\right)-\Delta\left(A^{-1}\right)\right] .
$$

При мальх и больших значениях $A$

$$
\overline{\mathbf{E}}_{\infty} \bar{\tau}_{g_{A}}^{*}= \begin{cases}\frac{A}{2}+O\left(A^{2}\right), & A \rightarrow 0 \\ A-\ln A+(1+\mathbb{C})+O\left(A^{-1} \ln ^{2} A\right), & A \rightarrow \infty\end{cases}
$$

4.3. Если требовать, чтобы момент $\bar{\tau}_{g_{A}}^{*}$ принадлежал классу $\overline{\mathfrak{M}}_{T}$ с некоторым $T>0$, то порог $A=A(T)$ следует выбирать из условия $\overline{\mathbf{E}}_{\infty} \bar{\tau}_{g_{A(T)}}^{*}=T$, т.е. в силу (48) определять из уравнения

$$
A-\left[F\left(A^{-1}\right)-\Delta\left(A^{-1}\right)\right]=T .
$$

Из (46) и (47) нетрудно итерациями заключить, что при малых и больших $T$ для $A(T)$ справедливы следуюшие асимптотики:

$$
A(T)= \begin{cases}2 T+O\left(T^{2}\right), & T \rightarrow 0, \\ T+\left[\ln T-(1+\mathbb{C})+O\left(T^{-1} \ln ^{2} T\right)\right], & T \rightarrow \infty .\end{cases}
$$




\section{5. Вычисление $\overline{\mathbf{E}}_{0} \bar{\tau}_{g_{A}}^{*}$ и оценки для $\bar{C}(T)$}

5.1. Справедлива следующая лемма.

Лемма 3. Для всякого $A>0$

$$
\overline{\mathbf{E}}_{0} \bar{\tau}_{g_{A}}^{*}=F\left(A^{-1}\right)-\Delta\left(A^{-1}\right) .
$$

При мальих и больших $A$

$$
\overline{\mathbf{E}}_{0} \bar{\tau}_{g_{A}}^{*}= \begin{cases}\frac{A}{2}+O\left(A^{2}\right), & A \rightarrow 0, \\ \ln A-(1+\mathbb{C})+O\left(A^{-1} \ln ^{2} A\right), & A \rightarrow \infty .\end{cases}
$$

Док аз а те льство. Согласно первому равенству в (23), $\overline{\mathbf{E}}_{0} \bar{\tau}_{g_{A}}^{*}=\int_{0}^{A} \mathbf{E}_{0} \tau_{A}^{(y)} g_{A}(y) d y$. В [11] найдено, что $\mathbf{E}_{0} \tau_{A}^{(y)}=F\left(A^{-1}\right)-F\left(y^{-1}\right)$, где $F(b)=e^{b}(-\operatorname{Ei}(-b))$. Значит, $\overline{\mathbf{E}}_{0} \bar{\tau}_{g_{A}}^{*}=F\left(A^{-1}\right)-\int_{0}^{A} F\left(y^{-1}\right) g_{A}(y) d y$.

По лемме 1

$$
g_{A}(y)=-\frac{1}{A y}+\frac{e^{-1 / y} \operatorname{Ei}\left(y^{-1}\right)}{A y^{2}}+\left(e^{1 / A}-A^{-1} \operatorname{Ei}\left(A^{-1}\right)\right) y^{-2} e^{-1 / y} .
$$

Поэтому, с учетом равенства $F\left(y^{-1}\right)=e^{1 / y}\left(-\operatorname{Ei}\left(-y^{-1}\right)\right)$, находим:

$$
\int_{0}^{A} F\left(y^{-1}\right) g_{A}(y) d y=-A^{-1} K_{1}+A^{-1} K_{2}+\left(e^{1 / A}-A^{-1} \operatorname{Ei}\left(A^{-1}\right)\right) K_{3},
$$

где

$$
\begin{gathered}
K_{1}=\int_{0}^{A} \frac{1}{y} e^{1 / y}\left(-\operatorname{Ei}\left(-y^{-1}\right)\right) d y, \quad K_{2}=\int_{0}^{A} y^{-2} \operatorname{Ei}\left(y^{-1}\right)\left(-\operatorname{Ei}\left(-y^{-1}\right)\right) d y \\
K_{3}=\int_{0}^{A} y^{-2}\left(-\operatorname{Ei}\left(-y^{-1}\right)\right) d y .
\end{gathered}
$$

Аналогично подсчетам для $J_{2}$ (см. (44)) находим, что

$$
K_{1}=-\int_{1 / A}^{\infty} \frac{e^{z}}{z} \operatorname{Ei}(-z) d z=\int_{0}^{\infty} e^{-z / A} \frac{\ln (1+z)}{z} d z
$$

Далее,

$$
\begin{aligned}
K_{2} & =-\int_{1 / A}^{\infty} \operatorname{Ei}(z) \operatorname{Ei}(-z) d z=-\int_{1 / A}^{\infty} \operatorname{Ei}(z) d\left(\operatorname{Ei}(-z) z+e^{-z}\right) \\
& =-\left.\operatorname{Ei}(z)\left(z \operatorname{Ei}(-z)+e^{-z}\right)\right|_{1 / A} ^{\infty}+\int_{1 / A}^{\infty}\left(e^{z} \operatorname{Ei}(-z)+\frac{1}{z}\right) d z .
\end{aligned}
$$

Аналогично (45) находим, что $\lim _{z \rightarrow \infty} \operatorname{Ei}(z)\left(z \operatorname{Ei}(-z)+e^{-z}\right)=0$. Учитывая представление $e^{z} \operatorname{Ei}(-z)+z^{-1}=\int_{0}^{\infty}(z+t)^{-2} e^{-t} d t$ (см. [2, формула 8.212 .3$])$, получаем, что

$$
\int_{1 / A}^{\infty}\left(e^{z} \operatorname{Ei}(-z)+z^{-1}\right) d z=-\operatorname{Ei}\left(-A^{-1}\right) e^{1 / A} .
$$


Таким образом,

$$
K_{2}=A^{-1} \operatorname{Ei}\left(A^{-1}\right) \operatorname{Ei}\left(-A^{-1}\right)+e^{-1 / A} \operatorname{Ei}\left(A^{-1}\right)-e^{1 / A} \operatorname{Ei}\left(-A^{-1}\right) .
$$

Наконец, $K_{3}=\int_{0}^{A} y^{-2}\left(-\operatorname{Ei}\left(-y^{-1}\right)\right) d y=-\int_{1 / A}^{\infty} \operatorname{Ei}(-z) d z=A^{-1} \operatorname{Ei}\left(-A^{-1}\right)+e^{-1 / A}$.

Из (50) и полученных представлений для $K_{1}, K_{2}$ и $K_{3}$ находим после простых подсчетов, что $\int_{0}^{A} F\left(y^{-1}\right) g_{A}(y) d y=\Delta\left(A^{-1}\right)$ и, значит,

$$
\overline{\mathbf{E}}_{0} \bar{\tau}_{g_{A}}^{*}=F\left(A^{-1}\right)-\int_{0}^{A} F\left(A^{-1}\right) g_{A}(y) d y=F\left(A^{-1}\right)-\Delta\left(A^{-1}\right) .
$$

Лемма 3 доказана.

5.2. Из (16), очевидной оценки $\bar{C}(T) \leqslant \overline{\mathrm{E}}_{0} \bar{\tau}_{g_{A}}^{*}$, где $A=A(T)$ выбирается так, чтобы было выполнено равенство $\overline{\mathbf{E}}_{\infty} \bar{\tau}_{g_{A(T)}^{*}}^{*}=T$, и лемм 2 и 3 получаем следующий основной результат настоящей работы.

Теорема. При любом $T>0$ минимаксныи риск

$$
\bar{C}(T)=\inf _{\bar{\tau} \in \overline{\mathfrak{M}}_{T}} \sup _{\theta \geqslant 0} \mathbf{E}_{\theta}(\bar{\tau}-\theta \mid \bar{\tau} \geqslant \theta)
$$

допускает следуюшие оченки сверху и снизу:

$$
F\left(T^{-1}\right)-\Delta\left(T^{-1}\right) \leqslant \bar{C}(T) \leqslant F\left(A^{-1}\right)-\Delta\left(A^{-1}\right)\left(=\bar{C}^{*}(T)\right),
$$

где $A=A(T)$ является корнем уравнения

$$
A-\left[F\left(A^{-1}\right)-\Delta\left(A^{-1}\right)\right]=T .
$$

При мальх T

$$
\frac{T}{2}+O\left(T^{2}\right) \leqslant \bar{C}(T) \leqslant T+O\left(T^{2}\right)
$$

При больших T

$$
\bar{C}(T)=\ln T-(1+\mathbb{C})+O\left(T^{-1} \ln ^{2} T\right),
$$

где $\mathbb{C}=0.577 \ldots-$ константа Эйлера. 


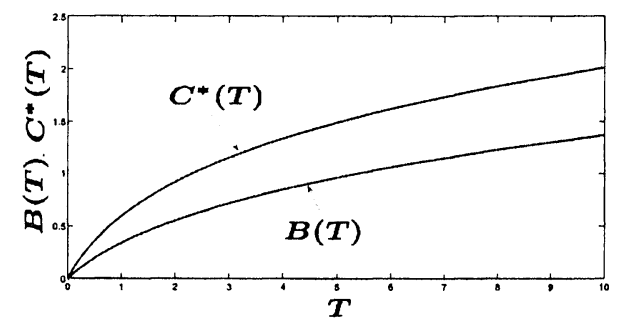

Рис. 1. Графики $C^{*}(T)-$ оценки сверху и $B(T)$ - оценки снизу минимаксного риска $C(T)$

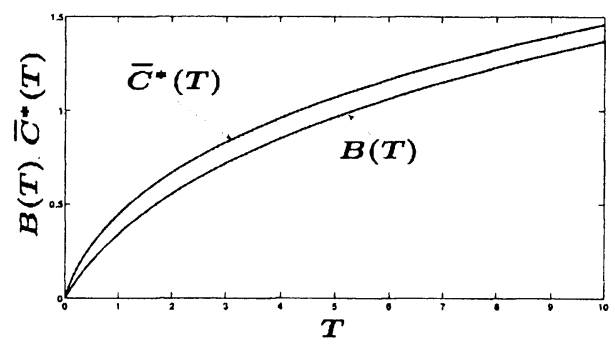

Рис. 2. Графики $\bar{C}^{*}(T)-$ оценки сверху и $B(T)$ - оценки снизу минимаксного риска $\bar{C}(T)$

Д о к а з а т е л в с т в о. Левое неравенство в (52) следует из того, что $B(T) \leqslant \bar{C}(T)$ и $B(T)=T / 2+O\left(T^{2}\right)$ (см. (9)). Правое неравенство в (52) вытекает из (51) и того, что при малых $A$ функция $F\left(A^{-1}\right)-$ $\Delta\left(A^{-1}\right)=B(A)$ ведет себя как $A / 2+O\left(A^{2}\right)$, а значит, в силу (47) при $A=A(T)$ и малых $T$

$$
\bar{C}(T) \leqslant A(T)-\frac{A(T)}{2}+O\left(A^{2}(T)\right)=2 T-\frac{2 T}{2}+O\left(T^{2}\right)=T+O\left(T^{2}\right) .
$$

Для доказательства (53) заметим, что, как было показано, $B(T) \leqslant$ $\bar{C}(T) \leqslant \overline{\mathbf{E}}_{0} \bar{\tau}_{g_{A(T)}}^{*}$. При больших $T$ из (9) находим

$$
B(T)=\ln T-1-\mathbb{C}+O\left(T^{-1} \ln ^{2} T\right) .
$$

В силу (49)

$$
\overline{\mathbf{E}}_{0} \bar{\tau}_{g_{A(T)}}^{*}=F\left(\frac{1}{A(T)}\right)-\Delta\left(\frac{1}{A(T)}\right)=B(A(T))
$$

При $T \rightarrow \infty$

$$
A(T)=T+\left[\ln T-1-\mathbb{C}+O\left(T^{-1} \ln ^{2} T\right)\right]
$$

и, следовательно,

$$
\begin{aligned}
\overline{\mathbf{E}}_{0} \bar{\tau}_{g_{A(T)}}^{*} & =B(A(T))=\ln A(T)-(1+\mathbb{C})+O\left(T^{-1} \ln ^{2} A(T)\right) \\
& =\ln T-(1+\mathbb{C})+O\left(T^{-1} \ln ^{2} T\right) .
\end{aligned}
$$

Из (54), (55) и (51) получаем требуемую асимптотику (53) для больших $T$. Теорема доказана.

На рис. 1 изображены графики $C^{*}(T)$ - оценки сверху и $B(T)-$ оценки снизу минимаксного риска $C(T)$. На рис. 2 изображены графики $\bar{C}^{*}(T)$ - оценки сверху и $B(T)$ - оценки снизу минимаксного риска $\bar{C}(T)$. 


\section{СПИСОК ЛИТЕРАТУРЫ}

1. Ватанабэ С., Икэда Н. Стохастические дифференциальные уравнения и диффузионные процессы. М.: Наука, 1986, 445 с.

2. Градштейн И. С., Рыжик И. М. Таблицы интегралов, сумм, рядов и произведений. М.: Физматгиз, 1971, 1108 с.

3. Лебедев Н.H. Специальные функции и их приложения. М.-Л.: Физматгиз, 1963, $358 \mathrm{c}$.

4. Jипцер P.Ш., Ширяев А.Н. Статистика случайных процессов. М.: Наука, 1974, $696 \mathrm{c}$.

5. Липчер Р. Ш., Ширяев А. Н. Теория мартингалов. М.: Наука, 1986, 512 с.

6. Ширяев $A . H$. Задача скорейшего обнаружения нарушения стационарного режима. - Докл. АН СССР, 1961, т. 138, № 5, с. 1039-1042.

7. Ширяев A.Н. Об оптимальных методах в задачах скорейшего обнаружения. Теория вероятн. и ее примен., 1963, т. 8, в. 1, с. 26-51.

8. Ширяев A.Н. Статистический последовательный анализ. М.: Наука, 1969, 231 с.

9. Cattiaux P., Collet P., Lambert A., Martínez S., Méléard S., San Martin J. Quasi-stationary distributions and diffusion models in population dynamics. arxiv: math/0703781v1

10. Collet P., Martínez S., San Martín J. Asymptotic laws for one-dimensional diffusions conditioned to nonabsorption. - Ann. Probab., 1995, v. 23, № 3, p. 1300-1314.

11. Feinberg E. A., Shiryaev A. N. Quickest detection of drift change for Brownian motion in generalized Bayesian and minimax settings. - Statist. Decisions, 2006, v. 24, p. $445-470$.

12. Kotz S., Nadarajah $S$. Extreme Value Distributions. London: Imperial College Press, 2000, 187 p.

13. Lladser M., San Martin J. Domain of attraction of the quasi-stationary distributions for the Ornstein-Uhlenbeck process. - J. Appl. Probab., 2000, v. 37, № 2, p. 511-520.

14. Mandl $P$. Spectral theory of semi-groups connected with diffusion processes and its application. - Czechoslovak Math. J., 1961, v. 11, № 4, p. 558-569.

15. Martínez S., San Martín J. Classification of killed one-dimensional diffusions. - Ann. Probab., 2004, v. 32, № 1A, p. 530-552.

16. Martínez S., San Martín J. Quasi-stationary distributions for a Brownian motion with drift and associated limit laws. - J. Appl. Probab., 1994, v. 31, № 4, p. 911-920.

17. Pinsky $R$. On the convergence of diffusion processes conditioned to remain in a bounded region for large time to limiting positive recurrent diffusion processes. Ann. Probab., 1985, v. 13, № 2, p. 363-378.

18. Pollak M., Siegmund D. Convergence of quasi-stationary to stationary distributions for stochastically monotone Markov processes. - J. Appl. Probab., 1986, v. 23, № 1, p. $215-220$.

19. Pollak M., Siegmund D. A diffusion process and its applications to detecting a change in the drift of Brownian motion. - Biometrika, 1985, v. 72, № 2, p. 267-280.

20. Pollak M. Optimal detection of a change in distribution. - Ann. Statist., 1985, v. 13, № 1, p. 206-227.

21. Ширяев $A . H$. От «разладки» к нелинейной фильтрации и теории мартингалов. Математические события ХХ века. М.: ФАЗИС, 2003, с. 491-518.

22. Shiryaev A.N. Quickest detection problems in the technical analysis of the financial data. - Mathematical Finance - Bachelier Congress (Paris, 2000). Berlin: Springer, 2002, p. 487-521.

23. Steinsaltz D., Evans S. N. Quasistationary distributions for one-dimensional diffusions with killing. - Trans. Amer. Math. Soc., 2007, v. 359, № 3, p. 1285-1324. 